\title{
Clinical characteristics and outcomes of end-stage renal disease patients with self-reported pruritus symptoms
}

This article was published in the following Dove Press journal: International Journal of Nephrology and Renovascular Disease 18 December 2013

Number of times this article has been viewed

\author{
Karthik Ramakrishnan' \\ T Christopher Bond' \\ Ami Claxton' \\ Vipan C Sood ${ }^{2}$ \\ Maria Kootsikas ${ }^{2}$ \\ Wendy Agnese ${ }^{2}$ \\ Scott Sibbel' \\ 'DaVita Clinical Research, \\ Minneapolis, MN, USA; ${ }^{2}$ Mitsubishi \\ Tanabe Pharma Corporation, Jersey \\ City, NJ, USA
}

\begin{abstract}
One of the most common conditions affecting end-stage renal disease (ESRD) patients undergoing hemodialysis (HD) is pruritus. Studies report that itchy and dry skin, symptoms of pruritus, affect $40 \%-90 \%$ of ESRD patients. Yet, in clinical practice the condition is often underdiagnosed resulting in inadequate management and an underappreciated impact on patient outcomes. Two retrospective analyses were conducted: a preliminary analysis of ESRD patients with pruritus symptoms $(n=73,124)$ undergoing HD or peritoneal dialysis at a large dialysis provider and a subsequent detailed analysis of a homogenous subset of patients undergoing in-center HD ( $\mathrm{n}=38,315)$. The goal was to better understand the clinical burden of pruritus as it relates to patient characteristics, quality of life, medication use, and HD compliance. This population is commonly burdened by multiple comorbidities and related polypharmaceutical management; identifying the relationship of pruritus to these ailments can help guide future research and resource allocation. The detailed analysis confirmed trends observed in the preliminary analysis: $30 \%$ reported being "moderately" to "extremely bothered" by itchiness. The HD patient population with the highest severity of self-reported pruritus also had a consistent trend in overall increased resource utilization - higher monthly doses of erythropoietin-stimulating agents (53,397.1 to 63,405.4 units) and intravenous (IV) iron (237.2 to 247.6 units) and higher use of IV antibiotics (14.1\% to $20.7 \%$ ), as well as poorer quality-of-life measures (25-point reductions in Burden of Disease Score and Effects on Daily Life subscales of the Kidney Disease Quality of Life-36 survey). These results highlight the need to better identify and manage ESRD patients impacted by pruritus, as this symptom is associated with negative clinical outcomes and increased resource utilization. Further studies are needed to evaluate the current economic burden of pruritus in ESRD patients and create possible options for an improved pharmacoeconomic profile in this patient population.
\end{abstract}

Keywords: pruritus, end-stage renal disease, hemodialysis, itchiness, patient reported outcomes

\section{Introduction}

Pruritus, a medical condition characterized by moderate to severe itchy and dry skin, affects a large proportion of end-stage renal disease (ESRD) patients undergoing hemodialysis (HD). Rates of reported pruritus among this population range from $40 \%-90 \%$ across various studies. Data from the Dialysis Outcomes and Practice Patterns Study demonstrated that $70 \%$ of HD patients experienced some pruritus, with $42 \%$ reporting moderate to extreme pruritus. ${ }^{1}$ The ITCH National Registry Study found that $84 \%$ of HD patients report pruritus, and $59 \%$ had it for more than a year. ${ }^{2}$ Research also indicates that pruritus increases with increasing severity of chronic kidney disease. ${ }^{3}$
Correspondence: Scott Sibbel Health Economics and Outcomes Research, DaVita Clinical Research, 825 South 8th Street, Suite 300, Minneapolis, MN 55404, USA

Tel +I 5 I5 2494444

Email scott.sibbel@davita.com 
The pathogenesis of pruritus in ESRD patients receiving HD is multifactorial. ${ }^{4,5}$ Different theories as to the cause of pruritus include metabolic disequilibrium, endocrine disorders, iron deficiency anemia, inadequate dialysate, peripheral nervous system neuropathies, immune system derangement, opioidergic system involvement, and histamine and serotonin involvement. ${ }^{2}$ Historically, researchers have explored various therapies to treat pruritus, with varying degrees of effectiveness. Currently, there are no United States Food and Drug Administration-approved therapies for uremic pruritus, and there are often inconsistencies when comparing the effectiveness of an intervention across studies. Limitations of these historical therapy studies include small sample sizes, lack of placebo control, and lack of blind and double-blind methodologies; thus, more rigorous studies are indicated. Currently, investigators are evaluating the efficacy of agonists/antagonists of the opioid system ${ }^{6,7}$ with gabapentin, pregabalin, and desloratadine, among others. ${ }^{8-14}$

Despite the prevalence of pruritus in the ESRD population, its clinical effects are poorly described in the literature. Some studies indicate that skin itchiness has negative effects on quality of life beyond discomfort, including fatigue, poor sleep quality, and depression. ${ }^{1,15,16}$ In addition, itchy skin may be associated with negative health outcomes and negative prognosis and mortality. ${ }^{1,17,18}$ For example, patients who report itchiness may have a higher rate of missed dialysis sessions as a consequence of their discomfort and may be at increased risk of hospitalization or death. ${ }^{2,19}$

This population is commonly burdened by multiple comorbidities and related polypharmaceutical management; identifying the relationship of pruritus to these ailments can help guide future research and resource allocation. The current analysis was undertaken to characterize the clinical burden of ESRD patients with pruritus symptoms receiving dialysis at a large dialysis organization (LDO). Our objective was to describe the clinical characteristics of ESRD patients by analyzing LDO data.

\section{Materials and methods}

Although pruritus is characterized by itchy and dry skin, the data reported here focus solely on itchiness. We conducted two retrospective cohort analyses: a preliminary overall patient analysis, including all dialysis patients (HD and peritoneal dialysis [PD]) and a detailed analysis looking at the outcomes of only in-center HD patients. The preliminary analysis was conducted to obtain an initial understanding of the clinical characteristics, quality of life, and medication use of ESRD patients reporting different itchiness severity.
The detailed analysis was conducted to get a more in-depth understanding regarding pruritus among HD patients only.

\section{Preliminary analysis}

The preliminary study population included all HD and PD patients with varying degrees of skin itchiness who received treatment at an LDO between January 2009 and May 2012 and who had responded to the Kidney Disease Quality of Life-36 (KDQOL) survey $(n=73,124)$. The KDQOL is a validated tool that assesses dialysis-specific, patient-reported, health-related quality-of-life findings, including itchy skin, for ESRD patients undergoing dialysis. ${ }^{20}$ Administration of the KDQOL is a Center for Medicare and Medicaid Services requirement that allows for the collection of information across many aspects of patients' experience with renal disease and dialysis therapy. The KDQOL is administered yearly to all dialysis patients at the LDO involved in this study.

Clinical outcomes included clinical patient characteristics, quality of life, and medication use. The quality-oflife analysis is specific to the KDQOL-SF (short form) 12 component scores (which summarize physical and mental components of dealing with kidney disease), the Burden of Disease subscale (which samples patients' impressions of kidney disease burden), the Symptoms and Problems subscale (which samples severity of symptoms), and the Effects on Daily Life subscale (which samples kidney disease effects) in relation to itchy skin.

Patients were grouped by their self-reported level of itchiness on the KDQOL survey scale (ordinals: 1, "not at all bothered"; 2, "somewhat bothered"; 3, "moderately bothered"; 4, "very much bothered"; 5 , "extremely bothered"). Patient clinical characteristics (ie, demographics, clinical laboratory results, comorbidities) were obtained and compared across the self-reported itchiness category using chi-square tests of trend for proportions and generalized linear model (GLM) tests for mean differences for all continuous outcomes.

To investigate quality-of-life measures, we tested for the association between itchiness severity on the KDQOL components: physical score, mental score, Effect on Daily Life subscale score, Burden of Disease subscale score, and Symptom and Problems subscale score. Although the preliminary overall sample population included 73,124 patients, we only included dialysis patients who completed the SF-12 portion of the KDQOL $(n=71,012)$. Analysis of variance (ANOVA) testing compared KDQOL component scores across the skin itchiness categories.

To investigate medication use, we analyzed intravenous (IV) antibiotic use, IV iron sucrose, and mean monthly 
erythropoietin-stimulating agent (ESA) dose by itchiness scores. From the overall sample, we identified patients $(n=70,500)$ who were given IV antibiotics within a 2-month window of KDQOL administration, IV iron sucrose, or ESA use. Tests for trend in IV antibiotics and IV iron use were performed via the Cochran-Armitage test. ANOVA analysis was performed to note significant differences in ESA utilization across itchiness severity categories.

\section{Detailed analysis}

The detailed analysis was conducted to further explore the results of the preliminary analyses, specifically among HD ESRD patients as the literature describes the high rates of pruritus among these patients. During this analysis, selfreported itchiness was analyzed. A retrospective cohort study was conducted using refined inclusion and exclusion criteria. This approach provided a homogenous treatment modality in the study population (ie, patients receiving in-center HD) that allowed for direct evaluation of HD adequacy and other modality specific measures.

Inclusion criteria included adult patients (age $\geq 18$ years), receiving in-center HD three times per week; receiving care in the LDO clinics between December 2008 and June 2012; completing a KDQOL survey after 3 months of dialysis therapy; and having Medicare as the primary payer. We kept the last KDQOL survey that patients took and made sure there was a 3-month dialysis period prior to those survey results $(\mathrm{n}=38,315)$. We excluded patients receiving PD, home HD, or nocturnal HD.

Study outcomes included measuring differences in patient characteristics, clinical laboratory values (serum phosphorous, corrected calcium, calcium phosphorous product $[\mathrm{Ca} * \mathrm{P}]$, serum parathyroid hormone $[\mathrm{PTH}]$, transferrin saturation, serum ferritin, hemoglobin [Hb], albumin, and $\mathrm{Kt} / \mathrm{V}$ ), medication use (IV ESAs, IV iron, IV vitamin D, IV antibiotics, cinacalcet hydrochloride, phosphate binders, and oral and topical pruritus medications), and HD treatment compliance (proportion of patients missing HD sessions) by skin itchiness severity.

Using the skin itchiness severity score from the last available KDQOL survey (defined as KDQOL index date) between January 2009 and September 2011, patients were grouped by severity score (ordinals: 1, "not at all bothered"; 2, "somewhat bothered"; 3, "moderately bothered"; 4, "very much bothered"; 5, "extremely bothered").

Patient clinical characteristics and clinical laboratory test values were generated and measured during the 1-month baseline period prior to the KDQOL index date and compared across the self-reported itchiness severity categories using chi-square tests of trend and GLM tests for mean differences.

Differences in medication utilization and HD compliance between skin itchiness severity categories were analyzed in the 6-month follow-up period after the KDQOL index date. Trends in IV medication use, information about dialysis sessions (both attended and missed), clinical laboratory testing, cinacalcet hydrochloride use, phosphate binder use, and pruritus medication use were descriptively assessed, and outcomes were summarized as rate of medication utilization, mean monthly medication dose, and mean number attended and missed HD sessions by itchiness severity.

Furthermore, GLM-mixed models, accounting for repeating measures over time, were fit to determine utilization differences for certain IV medications (ESA, iron, and vitamin D) at each month during the 6-month follow-up period. The outcomes of interest were mean cumulative monthly dose by itchiness severity category during each month of follow-up. The primary explanatory variable was the self-reported itchiness indicator; additional covariates included a follow-up month indicator and the interaction term of follow-up month and itchiness. Models were fit with a compound symmetry covariance structure, and both fixed effects and monthly means were provided. These models were further adjusted and included age, sex, race, dialysis vintage (years on dialysis), primary cause of ESRD, access type at baseline, index year (year of KDQOL index date), baseline comorbidities (cardiovascular disease, cancer, chronic obstructive pulmonary disease [COPD], liver disease), and history of transplant at baseline.

\section{Results \\ Preliminary analyses}

Six out of every 10 patients $(60 \%)$ in the preliminary analyses population reported some level of itching, with $14.5 \%$ of the patients reporting being "very much bothered" or "extremely bothered" by itchiness (Table 1). Statistical differences in patient characteristics were observed with increasing severity of self-reported itchiness. Patients, who self-reported higher degrees of itch were more likely to be younger and female with a clinically meaningful higher prevalence of certain comorbid conditions, including diabetes mellitus, cardiovascular disease, COPD, and liver disease. By itchiness, mean time on dialysis ranged from 3.55-3.72 years. While the majority of patients were Black and had diabetes as the cause of ESRD, no meaningful patterns in race or cause of ESRD were evident across itchiness severity categories. 
Table I Characteristics by itchiness score among all dialysis patients at a large dialysis organization (preliminary analysis)

\begin{tabular}{|c|c|c|c|c|c|c|}
\hline & \multicolumn{5}{|l|}{ Itchiness score } & \multirow{2}{*}{$\begin{array}{l}P \text {-value } \\
\text { for trend }\end{array}$} \\
\hline & $\mathbf{I}$ & 2 & 3 & 4 & 5 & \\
\hline Number of patients & $28,073(39.8 \%)$ & $21,320(30.2 \%)$ & $10,869(15.4 \%)$ & $6,521(9.3 \%)$ & $3,717(5.3 \%)$ & \\
\hline Age (years), mean (SD) & $60.34(14.83)$ & $60.11(14.55)$ & $60.0(14.62)$ & $59.58(14.57)$ & $58.51(14.38)$ & $<0.0001$ \\
\hline Time on dialysis (years), mean (SD) & $3.55(3.79)$ & $3.65(3.78)$ & $3.57(3.66)$ & $3.6 I(3.5 I)$ & $3.72(3.78)$ & 0.0201 \\
\hline Time on dialysis at LDO (years), mean (SD) & $2.91(2.99)$ & $2.98(2.97)$ & $2.96(2.95)$ & $2.97(2.86)$ & $2.98(2.95)$ & 0.0568 \\
\hline BMI $\left(\mathrm{kg} / \mathrm{m}^{2}\right)$, mean $(\mathrm{SD})$ & $28.98(7.60)$ & $29.09(7.67)$ & $29.19(7.72)$ & $29.03(7.88)$ & $29.20(8.12)$ & 0.0443 \\
\hline Female, \% & 44.82 & 44.07 & 43.44 & 47.22 & 49.96 & $<0.000$ I \\
\hline \multicolumn{7}{|l|}{ KDQOL component score* } \\
\hline Physical, mean (SD) & $39.27(10.67)$ & $36.96(10.15)$ & $34.89(9.66)$ & $33.14(9.30)$ & $32.12(9.47)$ & $<0.0001$ \\
\hline Mental, mean (SD) & $52.07(10.00)$ & $49.74(10.4 I)$ & $47.65(10.78)$ & $44.81(11.06)$ & $43.46(11.88)$ & $<0.0001$ \\
\hline \multicolumn{7}{|l|}{ Previous history, \% } \\
\hline Cardiovascular disease & 24.95 & 25.40 & 27.31 & 28.05 & 28.11 & $<0.0001$ \\
\hline Cancer & 2.09 & 2.04 & 2.28 & 2.33 & 2.02 & 0.3692 \\
\hline Chronic obstructive pulmonary disease & 3.01 & 3.46 & 4.05 & 4.68 & 5.43 & $<0.0001$ \\
\hline Diabetes & 53.30 & 53.27 & 54.89 & 56.83 & 56.36 & $<0.000$ I \\
\hline Liver disease & 1.38 & 1.72 & 1.68 & 1.75 & 1.99 & 0.0007 \\
\hline
\end{tabular}

Notes: *Includes only patients with complete SF-I2 (all 12 questions-non 0 score); $n=68,426$. Itchiness scores are I, "not at all bothered"; 2 , "somewhat bothered"; 3 , "moderately bothered"; 4, "very much bothered"; 5 , "extremely bothered." The total number of patients included in this table is 70,500, which is a subset of patients who were given IV antibiotics within a 2-month window of KDQOL administration, IV iron sucrose, or ESA use; no data imputation was used.

Abbreviations: BMI, body mass index; ESA, erythropoietin-stimulating agent; IV, intravenous; KDQOL, Kidney Disease Quality of Life; LDO, large dialysis organization; $\mathrm{SD}$, standard deviation.

For laboratory results (Table 2), statistically significant differences by itchiness severity were found for $\mathrm{Hb}$ $(P=0.0361)$ and for phosphorus, calcium, PTH, serum ferritin, and albumin $(P<0.0001$ for each).

Increasing skin itchiness severity was associated with lower quality-of-life scores on the KDQOL-36 survey. A statistically significant decreasing trend in physical score (39.27 to 32.12, $P<0.0001$ ) and mental score (52.07 to 43.46, $P<0.0001)$ was observed, respectively, from the lowest to the highest itchiness severity category (Figure 1 and Table 1). Similar trends were observed for the Effect on Daily Life subscale score ( 76.40 to $51.85, P<0.0001)$, Burden of Disease subscale score (57.23 to 32.71, $P<0.0001$ ), and Symptom and Problem subscale scores (85.73 to 52.26) (Table 3). Summary statistics provided for the Symptom and Problems subscale scores showed a substantially decreasing symptom score with increasing severity of itch; however, this trend could not be tested for statistical significance as itchiness severity questions were included in computing KDQOL Symptom and Problems subscale score, and itchiness could not be included as independent variables in the ANOVA analysis (Table 3).

With regard to medication use, IV antibiotic use increased with itchiness severity across HD access types (Figure 2). The highest reported IV antibiotic use rates were found among those patients with a central venous catheter $(n=12,004)$; these patients reported antibiotic use ranging from $21.8 \%$ in the "not at all bothered" itch category to $28.5 \%$ in the "extremely bothered" itch category $(P<0.0001)$. Patients with a PD catheter $(\mathrm{n}=6,705)$ reported IV antibiotic use rates ranging from $9.1 \%$ to $11.6 \%$, those with an arteriovenous graft $(n=12,868)$ ranged from $6.5 \%$ to $9.0 \%$, and those with an arteriovenous fistula ( $\mathrm{n}=38,899$ ) ranged from $5.1 \%$ to $7.3 \%$ (all $P<0.05$ ). Among patients receiving an ESA, the mean monthly ESA dose rose

Table 2 Baseline dialysis-related clinical measures by itchiness score among all dialysis patients at a large dialysis organization (preliminary analysis)

\begin{tabular}{|c|c|c|c|c|c|c|}
\hline & \multicolumn{5}{|l|}{ Itchiness score } & \multirow{2}{*}{$\begin{array}{l}P \text {-value } \\
\text { for trend }\end{array}$} \\
\hline & I & 2 & 3 & 4 & 5 & \\
\hline Phosphorus, mmol/L (SD) & $1.6 \mathrm{I}(0.43)$ & $1.66(0.45)$ & $\mathrm{I} .67(0.47)$ & $1.69(0.49)$ & I.7I $(0.50)$ & $<0.0001$ \\
\hline Calcium, mmol/L (SD) & $2.27(0.16)$ & $2.28(0.16)$ & $2.28(0.17)$ & $2.27(0.17)$ & $2.29(0.17)$ & $<0.000 \mathrm{I}$ \\
\hline PTH, ng/L (SD) & $4 \mid 4.77(318.54)$ & $426.5 \mathrm{I}(336.5 \mathrm{I})$ & $423.78(330.50)$ & $44 I .25(346.56)$ & $439.61(348.30)$ & $<0.0001$ \\
\hline TSAT, \% (SD) & $31.42(13.84)$ & $31.61(14.05)$ & $31.42(13.95)$ & $31.42(14.44)$ & $30.76(13.87)$ & 0.1394 \\
\hline Serum ferritin, pmol/L (SD) & I,52I.29 (858.I7) & $\mathrm{I}, 5 \mathrm{I} 3.06(85 \mathrm{I} .86)$ & $1,500.30(847.64)$ & I,489.87 (875.54) & $\mathrm{I}, 448.62(87 \mathrm{I} .84)$ & $<0.0001$ \\
\hline Hemoglobin, mmol/L (SD) & $6.72(0.69)$ & $6.73(0.78)$ & $6.72(0.69)$ & $6.70(0.72)$ & $6.69(0.7 \mathrm{I})$ & 0.0361 \\
\hline Albumin, g/L (SD) & $39.40(4.00)$ & $39.30(4.00)$ & $39.20(4.00)$ & $38.90(4.20)$ & $38.50(4.40)$ & $<0.0001$ \\
\hline
\end{tabular}

Notes: Itchiness scores are I, "not at all bothered"; 2, "somewhat bothered"; 3, "moderately bothered"; 4, "very much bothered”; 5, "extremely bothered." Abbreviations: PTH, parathyroid hormone; SD, standard deviation; TSAT, transferrin saturation. 
Itchiness score

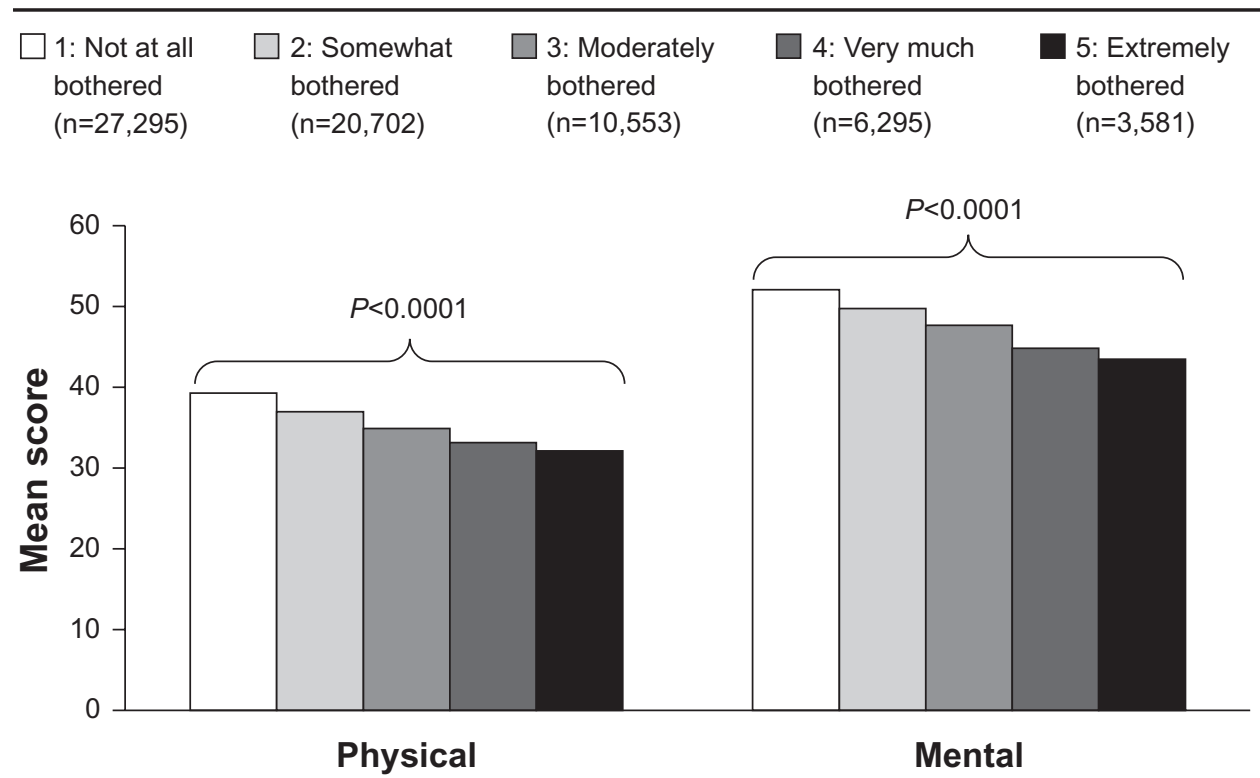

Figure I Kidney disease quality of life assessment: physical and mental component scores by itchiness (preliminary analysis).

Note: The total number of patients included in this figure is a subset of patients with complete responses for the physical and mental component of the KDQOL survey; no data imputation was used.

Abbreviation: KDQOL, Kidney Disease Quality of Life.

(55,653.03 units for "not at all bothered"; $66,212.51$ units for "extremely bothered," $P<0.0001)$ with increased itchiness (Figure 3). Furthermore, approximately $75 \%$ of the study population received IV iron sucrose; while the mean monthly dose rose with increased itchiness (257.84 units for "not at all bothered"; 262.55 units for "extremely bothered").

\section{Detailed analyses}

Demographic and clinical characteristics of the study population in the detailed analysis $(n=38,315)$ were consistent with the study population from the preliminary analysis (Tables 4 and 5). In the detailed analysis, $15 \%$ of the total population reported being "very much bothered" or "extremely bothered" by itchiness, and this proportion increased to $30 \%$ when the "moderately bothered" category was included.

Across patient characteristics for this study population, modest, yet statistically significant differences were seen with increasing levels of skin itchiness ("not at all bothered" to "extremely bothered") for age, sex, race, diabetes-caused ESRD, and dialysis vintage. Increasing skin itchiness $(P<0.05)$ was reported by younger patients (63.32 to 59.37 years), women ( $46.3 \%$ to $50.9 \%$ ), Blacks ( $41.9 \%$ to $45.6 \%$ ), patients with diabetes as the cause of their ESRD (44.1\% to 43.9\%), and those who spent more time on dialysis (3.83 to 4.07 years).

In comparison to the preliminary analysis, larger comorbidity burden was observed. For the detailed analysis, the range for cardiovascular disease was $28.5 \%$ to $32.7 \%$, COPD was $3.4 \%$ to $6.5 \%$, and liver disease was $1.8 \%$ to $2.7 \%(P<0.01$ for all $)$ across skin itchiness categories ("not at all bothered" to "extremely bothered"). In addition, patients were more likely to have infection-related conditions, including septicemia (27.4\% to $33.9 \%$, $P<0.0001)$ and bacteremia (5.9\% to $7.9 \%, P<0.0001)$.

Statistically significant differences were observed in clinical laboratory measures (serum phosphorous, corrected

Table 3 Association between itchiness and KDQOL symptom, burden, and effects subscales scores among all dialysis patients at a large dialysis organization (preliminary analysis)

\begin{tabular}{|c|c|c|c|c|c|c|}
\hline & \multicolumn{5}{|c|}{ Itchiness score } & \multirow{2}{*}{$\begin{array}{l}P \text {-value for } \\
\text { ANOVA }\end{array}$} \\
\hline & 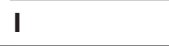 & 2 & 3 & 4 & 5 & \\
\hline Number of patients & 29,158 & 22,108 & 11,237 & 6,780 & $3,84 I$ & \\
\hline Symptom score, mean (SD) & 85.73 (II.69) & 78.48 (II.9I) & $69.21(13.16)$ & $60.45(14.94)$ & $52.26(17.90)$ & NA \\
\hline Burden score, mean (SD) & $57.23(29.60)$ & $50.04(28.58)$ & $43.84(27.33)$ & $36.99(26.27)$ & $32.71(27.75)$ & $<0.0001$ \\
\hline Effects score, mean (SD) & $76.40(20.86)$ & $70.17(21.50)$ & 63.01 (22.01) & $57.92(23.08)$ & $51.85(25.30)$ & $<0.0001$ \\
\hline
\end{tabular}

Notes: Itchiness scores are I, "not at all bothered"; 2, "somewhat bothered"; 3, "moderately bothered"; 4, "very much bothered"; 5, "extremely bothered." Includes patients with valid subscales (algorithm allows for missing questions).

Abbreviations: ANOVA, analysis of variance; NA, not applicable; SD, standard deviation; KDQOL, Kidney Disease Quality of Life. 


\section{Itchiness score}

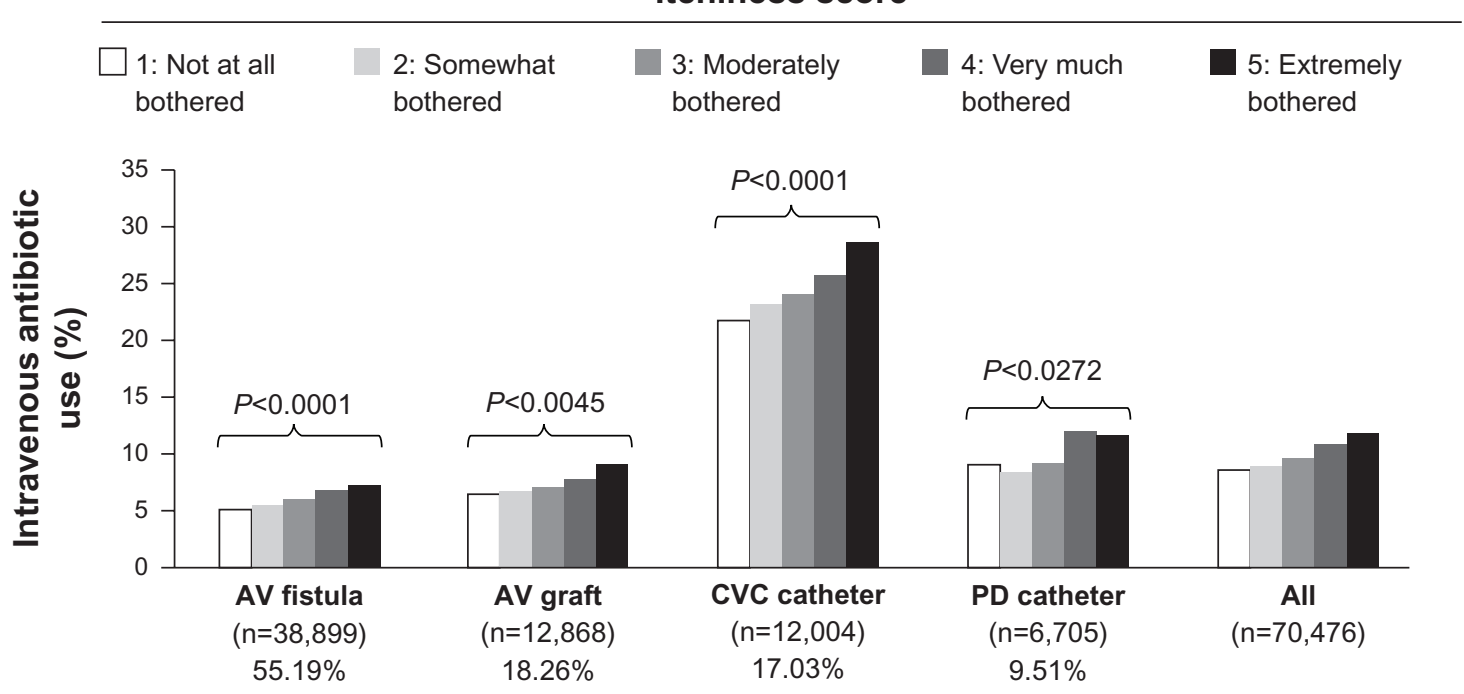

Figure 2 Intravenous antibiotic use by itchiness and type of access (preliminary analysis).

Notes: This figure shows intravenous antibiotic use of patients who self-reported itchiness was higher for patients who have a central venous catheter (CVC) than other access types. The $P$-value represents the difference between a category of itchiness score compared to those who reported being extremely bothered by itchiness (category 5 ). Overall $P$-value was not determined for multiple comparisons. The total number of patients included in this figure represent those patients for whom we had complete data regarding dialysis access; no data imputation was used.

Abbreviations: AV, arteriovenous; PD, peritoneal dialysis.

calcium, $\mathrm{Ca} * \mathrm{P}$, and albumin); however, most of these differences were small in magnitude as in the preliminary analysis (Table 5).

In the detailed analysis, an increase in medication utilization and missed HD sessions were observed with increasing itchiness severity (Tables 6 and 7). Across the itchiness categories ("not at all bothered" to "extremely bothered"), patients were more likely to use higher monthly doses of an
ESA (53,397.1 to 63,405.4), IV iron (237.2 to 247.6$)$, and IV antibiotics (14.1\% to $20.7 \%$ ). Patients were also more likely to use more antipruritic medications (eg, IV, topical, oral; $20.9 \%$ to $40.8 \%)$, IV diphenhydramine (1.4\% to $6.0 \%)$ with average number of administration ranging from 20.2 to 30.9 per month, topical medications ( $2.9 \%$ to $4.5 \%)$, and oral medications (13.2\% to 30.6\%). For other medications (eg, IV vitamin $\mathrm{D}$, cinacalcet hydrochloride, phosphate binders),

Itchiness score

\begin{tabular}{lllll}
\hline$\square$ 1: Not at all & 2: Somewhat & 3: Moderately & 4: Very much & 5: Extremely \\
bothered & bothered & bothered & bothered & bothered \\
$(n=27,295)$ & $(n=20,702)$ & $(n=10,553)$ & $(n=6,295)$ & $(n=3,581)$
\end{tabular}

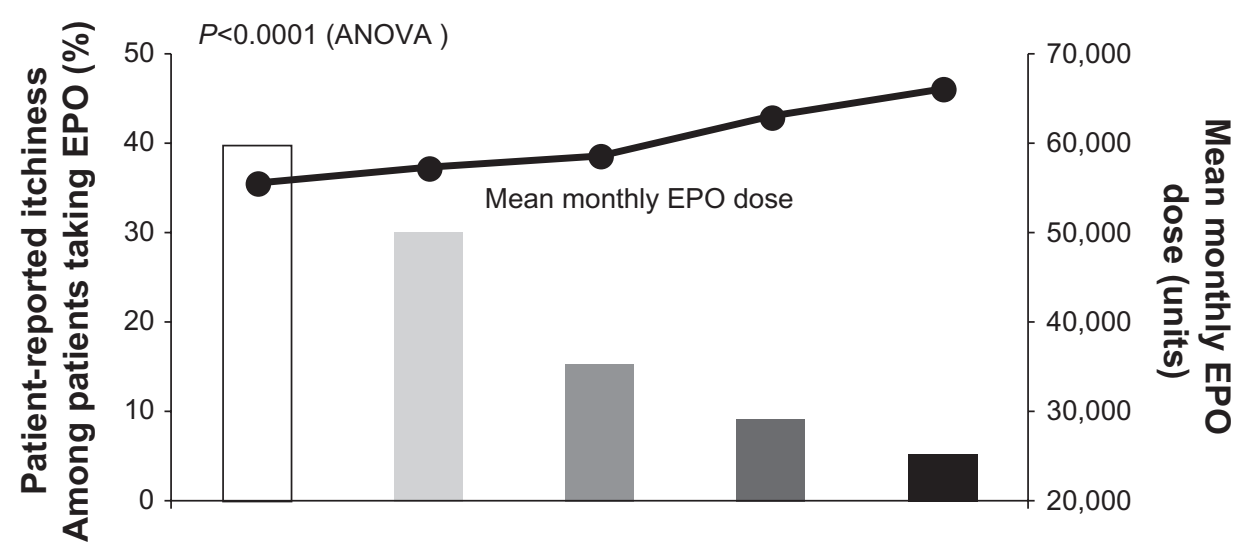

Figure 3 EPO administration in dialysis patients by itchiness score (preliminary analysis).

Notes: This figure shows the number of dialysis patients ( $n=68,426)$ who were receiving EPO (left $y$ axis). The mean monthly dose of EPO (right $y$ axis) shows that among patients receiving EPO, the mean monthly EPO dose rose with increased itchiness.

Abbreviations: ANOVA, analysis of variance; EPO, Epogen ${ }^{\circledR}$. 
Table 4 Characteristics by itchiness score among hemodialysis patients at a large dialysis organization (detailed analysis)

\begin{tabular}{|c|c|c|c|c|c|c|}
\hline & \multicolumn{5}{|l|}{ Itchiness score } & \multirow{2}{*}{$\begin{array}{l}P \text {-value } \\
\text { for trend }\end{array}$} \\
\hline & I & 2 & 3 & 4 & 5 & \\
\hline Number of patients & $15,319(40.0 \%)$ & I I,567 (30.2\%) & $5,867(15.3 \%)$ & $3,571(9.3 \%)$ & $\mathrm{I}, 99 \mathrm{I}(5.2 \%)$ & \\
\hline Age (years), mean (SD) & $62.32(14.44)$ & $61.96(14.25)$ & $61.55(14.3)$ & $61.32(14.61)$ & $59.37(14.25)$ & $<0.0001$ \\
\hline Time on dialysis (years), mean (SD) & $3.83(3.85)$ & $4.01(3.89)$ & $3.98(3.89)$ & $3.9(3.56)$ & $4.07(3.88)$ & 0.004 \\
\hline Time on dialysis at LDO (years), mean (SD) & $3.08(3.06)$ & $3.2(3.03)$ & $3.19(3.05)$ & $3.17(2.94)$ & $3.19(3.03)$ & 0.0155 \\
\hline BMI $\left(\mathrm{kg} / \mathrm{m}^{2}\right)$, mean $(\mathrm{SD})$ & $28.76(7.10)$ & $28.9(7.16)$ & $29.06(7.46)$ & $28.85(7.33)$ & $28.93(7.62)$ & 0.0978 \\
\hline Female, \% & 46.3 & 45.47 & 45.36 & 48.19 & 50.88 & 0.0022 \\
\hline \multicolumn{7}{|l|}{ Previous history, \% } \\
\hline Cardiovascular disease & 28.53 & 29.45 & 31.36 & 31.50 & 32.70 & $<0.0001$ \\
\hline Cancer & 2.21 & 2.43 & 2.49 & 2.80 & 2.71 & 0.0215 \\
\hline Chronic obstructive pulmonary disease & 3.39 & 4.05 & 4.57 & 5.26 & 6.48 & $<0.0001$ \\
\hline Liver disease & 1.81 & 2.03 & 2.03 & 2.38 & 2.66 & 0.0029 \\
\hline Bacteremia & 27.39 & 29.20 & 31.57 & 32.68 & 33.85 & $<0.0001$ \\
\hline Septicemia & 5.88 & 6.57 & 7.12 & 7.20 & 7.89 & $<0.0001$ \\
\hline Race/ethnicity, \% & & & & & & $<0.0001$ \\
\hline Black & 41.94 & 41.71 & 39.34 & 41.89 & 45.61 & \\
\hline Asian & 1.88 & 2.12 & 2.44 & 2.63 & 1.46 & \\
\hline Caucasian & 37.57 & 37.82 & 39.82 & 36.26 & 34.2 & \\
\hline Hispanic & 14.28 & 13.74 & 13.87 & 15.23 & 14.97 & \\
\hline Other & 4.33 & 4.61 & 4.53 & 3.98 & 3.77 & \\
\hline Cause of ESRD, \% & & & & & & 0.0339 \\
\hline Diabetes & 44.10 & 43.43 & 43.43 & 45.53 & 43.95 & \\
\hline Hypertension & 32.30 & 32.84 & 31.63 & 30.52 & 30.29 & \\
\hline Other & 23.60 & 23.73 & 24.94 & 23.94 & 25.77 & \\
\hline
\end{tabular}

Notes: Itchiness scores are I, "not at all bothered"; 2, "somewhat bothered"; 3, "moderately bothered"; 4, "very much bothered"; 5, "extremely bothered." Abbreviations: BMI, body mass index; ESRD, end-stage renal disease; LDO, large dialysis organization; SD, standard deviation.

while an increasing trend was noted, differences in utilization were not deemed clinically meaningful.

When ESA utilization was modeled across itchiness severity categories during each month of follow up, statistically significant differences were observed over time and between categories $(P<0.0001)$. Overall ESA utilization decreased across the 6-month observation for all severity categories. Despite the overall decline, increasing ESA utilization was observed across the itchiness categories within each month of the 6-month observation period.
An approximate 5,000-unit increase in monthly ESA dose was observed across the itchiness categories (Figure 4).

Though many of the IV iron and vitamin D utilization differences between itchiness severity categories were statistically significant, the numerical differences were small, suggesting that these differences may not be clinically meaningful (Figure 5; Tables 6 and 7).

Patients with increasing itchiness severity were less compliant with HD treatment (ie, more likely to miss HD sessions). An increase in the mean number of missed sessions

Table 5 Baseline dialysis-related clinical measures by itchiness score among hemodialysis patients at a large dialysis organization (detailed analysis)

\begin{tabular}{|c|c|c|c|c|c|c|}
\hline & \multicolumn{5}{|l|}{ Itchiness score } & \multirow{2}{*}{$\begin{array}{l}P \text {-value } \\
\text { for trend }\end{array}$} \\
\hline & I & 2 & 3 & 4 & 5 & \\
\hline $\begin{array}{l}\text { Serum phosphorus, mmol/L, mean } \\
\text { (SD) }\end{array}$ & $1.59(0.48)$ & $1.64(0.5 \mathrm{I})$ & $1.65(0.51)$ & $1.67(0.55)$ & I.7I (0.55) & $<0.0001$ \\
\hline $\begin{array}{l}\text { Corrected calcium, mmol/L, mean } \\
\text { (SD) }\end{array}$ & $2.18(0.45)$ & $2.19(0.45)$ & $2.20(0.4 I)$ & $2.19(0.44)$ & $2.22(0.40)$ & $<0.0001$ \\
\hline Ca*P, mean (SD) & $3.54(1.16)$ & $3.67(1.25)$ & $3.70(1.25)$ & $3.71(1.34)$ & $3.83(1.36)$ & $<0.0001$ \\
\hline Serum PTH, ng/L, mean (SD) & $350.45(368.28)$ & $362.35(372.45)$ & $358.45(356.37)$ & $375.08(376.29)$ & $368.92(367.93)$ & 0.0003 \\
\hline TSAT, \%, mean (SD) & $26.63(16.88)$ & $26.92(16.81)$ & $26.75(16.79)$ & $26.60(16.60)$ & $26.90(16.92)$ & 0.6500 \\
\hline Serum ferritin, pmol/L, mean (SD) & I,I 61.43 (970.79) & I,I 67.74 (948.9I) & I,I $67.59(957.22)$ & I, I $78.73(1,005.40)$ & I, I I 4.38 (926.33) & 0.5620 \\
\hline Hemoglobin, mmol/L, mean (SD) & $6.81(0.73)$ & $6.83(0.88)$ & $6.83(0.74)$ & $6.80(0.77)$ & $6.80(0.78)$ & 0.8122 \\
\hline Albumin, g/L, mean (SD) & $38.30(7.50)$ & $38.30(7.50)$ & $38.40(7.30)$ & $37.80(7.90)$ & $37.70(7.30)$ & 0.0001 \\
\hline $\mathrm{Kt} / \mathrm{V}$, mean (SD) & I.56 (0.52) & I.56 (0.50) & I.57 (0.49) & I.55 (0.5I) & $\mathrm{I} .56(0.49)$ & 0.7504 \\
\hline
\end{tabular}

Notes: Itchiness scores are I, "not at all bothered"; 2, "somewhat bothered"; 3, "moderately bothered"; 4, "very much bothered"; 5, "extremely bothered."

Abbreviations: Ca*P, calcium phosphorous product; PTH, parathyroid hormone; SD, standard deviation; TSAT, transferrin saturation. 
Table 6 Adjusted intravenous medication utilization by itchiness score among hemodialysis patients at a large dialysis organization (detailed analysis)

\begin{tabular}{|c|c|c|c|c|c|c|}
\hline & \multicolumn{6}{|l|}{ Itchiness } \\
\hline & $\begin{array}{l}\text { Not at all } \\
\text { bothered } \\
n=15,319\end{array}$ & $\begin{array}{l}\text { Somewhat } \\
\text { bothered } \\
n=11,567\end{array}$ & $\begin{array}{l}\text { Moderately } \\
\text { bothered } \\
n=5,867\end{array}$ & $\begin{array}{l}\text { Very much } \\
\text { bothered } \\
n=3,57 \text { I }\end{array}$ & $\begin{array}{l}\text { Extremely } \\
\text { bothered } \\
n=I, 99 \mid\end{array}$ & $\begin{array}{l}P \text {-value } \\
\text { for fixed } \\
\text { effect }\end{array}$ \\
\hline EPO utilization (units) & & & & & & $<0.000 I^{a}$ \\
\hline Month I & $44,954.22$ & $44,990.20$ & $45,720.4 I$ & $47,729.26$ & $50,700.95$ & $<0.000 \mathrm{I}^{\mathrm{b}}$ \\
\hline Month 2 & $43,932.08$ & $44,587.11$ & $45,125.37$ & $47,557.75$ & $50,106.20$ & \\
\hline Month 3 & $43,490.60$ & $44,108.16$ & $44,578.19$ & $46,882.51$ & $48,776.31$ & \\
\hline Month 4 & $42,463.49$ & $43,100.94$ & $43,730.45$ & $45,862.36$ & $46,887.20$ & \\
\hline Month 5 & $40,814.80$ & $41,556.12$ & $42,327.83$ & $44,954.22$ & $45,387.86$ & \\
\hline Month 6 & $40,570.64$ & $40,810.72$ & $41,701.82$ & $43,875.00$ & $45,066.75$ & \\
\hline Iron utilization (mg) & & & & & & $0.0314^{\mathrm{a}}$ \\
\hline Month I & 201.30 & 198.68 & 196.19 & 206.19 & 199.26 & $<0.000 \mathrm{I}^{\mathrm{b}}$ \\
\hline Month 2 & 200.36 & 198.26 & 198.98 & 200.60 & 203.73 & \\
\hline Month 3 & 204.20 & 202.39 & 204.18 & 207.04 & 212.96 & \\
\hline Month 4 & 199.08 & 200.88 & 200.42 & 204.40 & 207.68 & \\
\hline Month 5 & 196.02 & 196.68 & 194.09 & 197.14 & 199.42 & \\
\hline Month 6 & 199.32 & 198.84 & 199.26 & 200.64 & 205.98 & \\
\hline Vitamin D (mg) & & & & & & $0.0066^{\mathrm{a}}$ \\
\hline Month I & 39.84 & 40.97 & 41.78 & 41.17 & 43.00 & $<0.000 \mathrm{I}^{\mathrm{b}}$ \\
\hline Month 2 & 40.99 & 42.01 & 42.48 & 42.03 & 43.33 & \\
\hline Month 3 & 41.71 & 42.81 & 43.63 & 42.27 & 44.12 & \\
\hline Month 4 & 42.56 & 43.73 & 43.75 & 43.13 & 44.46 & \\
\hline Month 5 & 43.12 & 44.38 & 44.90 & 43.52 & 46.09 & \\
\hline Month 6 & 44.39 & 45.21 & 45.80 & 45.00 & 45.77 & \\
\hline
\end{tabular}

Notes: Measures of variability have not been added due to the log transformation of the outcome variable, which may be subject to back transformation bias. ${ }^{\text {a Represents }}$ $P$-values for the analysis across itchiness scores; ${ }^{b}$ represents $P$-values for the analysis across months.

Abbreviation: EPO, Epogen ${ }^{\circledR}$.

per month was observed as itchiness increased ( 0.60 to 0.83 ). The proportion of patients with missed dialysis sessions ranged from $56.16 \%$ ("not at all bothered" category) to $63.23 \%$ ("extremely bothered" category). Among patients who missed dialysis sessions, patients with the most severe itchiness missed on average 2.6 more dialysis sessions per year compared to patients with no itchiness (Table 8).

\section{Discussion}

A preliminary analysis of a dialysis (mixed-modality) population from a LDO found that $60 \%$ of all patients report some level of skin itchiness, which is consistent with previously reported prevalence data. ${ }^{1,2}$ The perception of itch as self-reported on a validated assessment tool was associated with quality-of-life factors and clinical factors. Clinical characteristics and outcomes of patients were generally consistent across the various itchiness severity categories.

For the patients in the preliminary analysis, modest, yet statistically significant differences were seen with increasing levels of skin itchiness across patient characteristics of age, sex, race, and ethnicity, cause of ESRD, and dialysis vintage (Table 1). Differences were also observed in the comorbidity burden, vascular access, attended sessions, mean clinical laboratory values (phosphorous, corrected calcium, $\mathrm{Ca}^{*} \mathrm{P}, \mathrm{PTH}$, serum ferritin, albumin, $\mathrm{Hb}$ ), and use of pruritus treatments. For patients reporting the most severe itchiness, larger differences existed in two categories: percent use of antibiotics and use of pruritus treatments. An association was also found between itchiness severity and ESA use.

Increasing itch severity, as measured using validated component and subscale scores on the KDQOL survey, was associated with reduced quality of life. Patients who were "extremely bothered" versus "not at all bothered" by itch severity reported a 7-point reduction in physical component score, 9-point reduction in mental component scores, 25-point reduction in Burden of Disease scores, and 25-point reduction in Effects on Daily Life subscales. Each item on the KDQOL SF-12 survey is scored on a 100-point scale, and items are averaged in each scale. Studies have shown that improvements in KDQOL scores are related to reductions in hospitalization and mortality; Lowrie and Lew found that a 1-point increase in either the physical component score or the mental component score is related to a $2 \%$ decrease in mortality and a $2 \%$ decrease in hospitalization. ${ }^{21}$ In another study, a 10-point decrease in individual patient scores over 


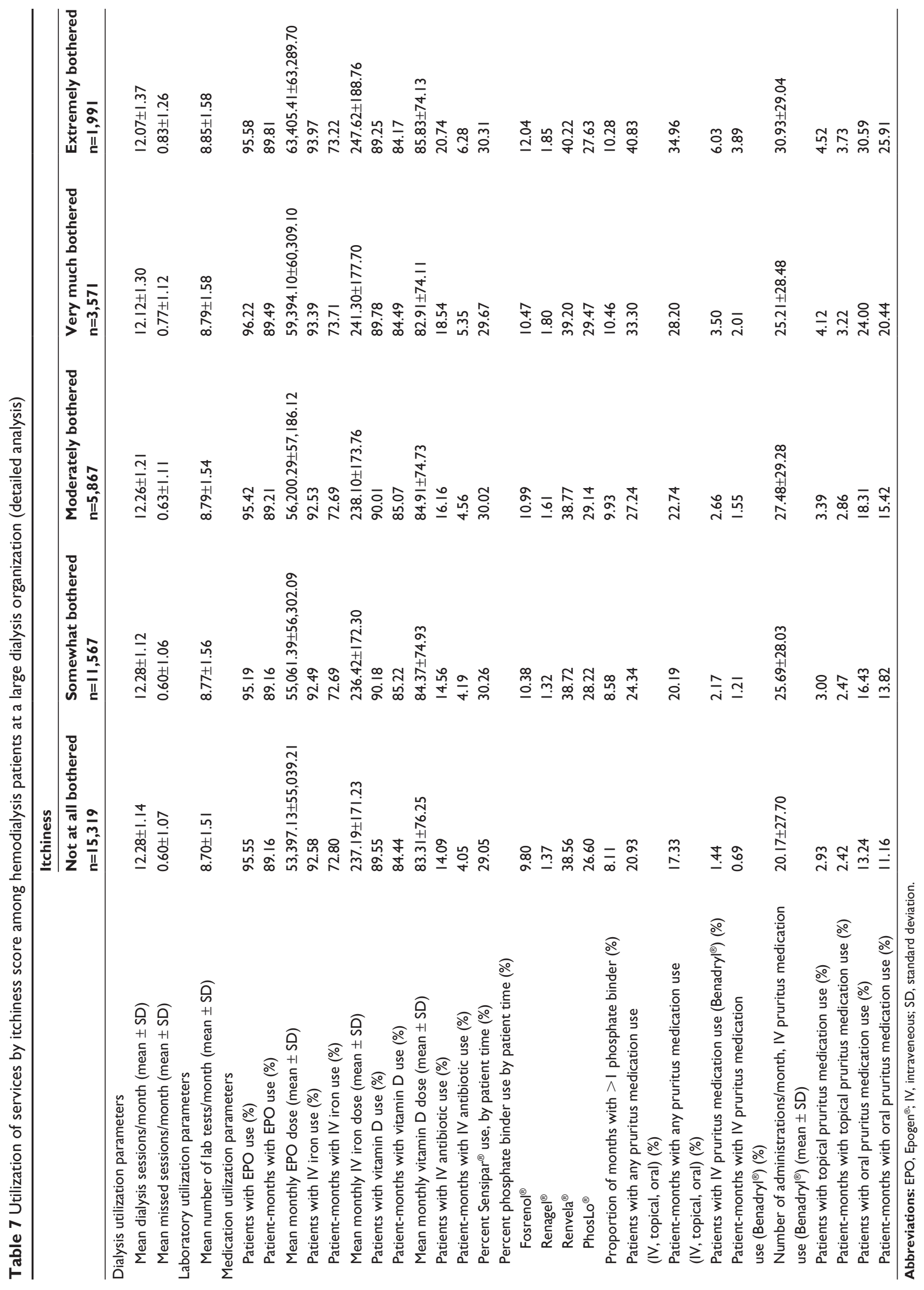


Table 8 Missed dialysis sessions in the 6 months following the KDQOL survey

\begin{tabular}{lllll}
\hline & & \multicolumn{3}{c}{ Itchiness score } \\
\cline { 2 - 5 } & $\mathbf{I}$ & $\mathbf{2}$ & $\mathbf{3}$ & $\mathbf{4}$ \\
\hline Number of patients & 15,319 & $\mathrm{II}, 567$ & 5,867 & $3,57 \mathrm{I}$ \\
Missed sessions (\%) & 56.16 & 56.52 & 56.93 & $3.99 \mathrm{I}$ \\
Missed sessions, mean (SD) & $3.6 \pm 6.4$ & $3.5 \pm 6.3$ & $3.8 \pm 6.6$ & $4.5 \pm 7.2$ \\
\hline
\end{tabular}

Notes: Increasing score means increased itchiness (I, "not at all bothered"; 2, "somewhat bothered"; 3, "moderately bothered”; 4, "very much bothered"; 5, "extremely bothered").

Abbreviations: SD, standard deviation; KDQOL, Kidney Disease Quality of Life.

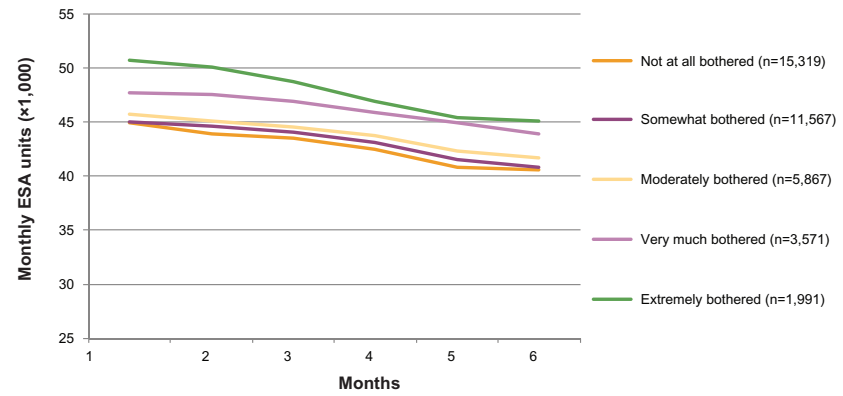

Figure 4 Monthly erythropoiesis-stimulating agent (ESA) utilization differences by itchiness score (detailed analysis).

time significantly increased the risk of hospitalization and death. ${ }^{22}$

Because self-reported skin itchiness was associated with increased clinical burden in a population with mixed dialysis modalities, a rigorous retrospective cohort study was conducted using refined inclusion and exclusion criteria that represented a population of ESRD patients receiving in-center HD. This approach provided a homo-genous study population and allowed for direct evaluation of HD adequacy and other modality-specific measures and included selfreported itchiness. Among this population, 30\% reported moderate to severe skin itchiness. Trends in patient characteristics and comorbidities were similar to those trends found in the preliminary analysis; such results are consistent with individuals being immunocompromised and susceptible to infections. For instance, clinically meaningful differences in comorbidity burden were found among the itchiness strata,

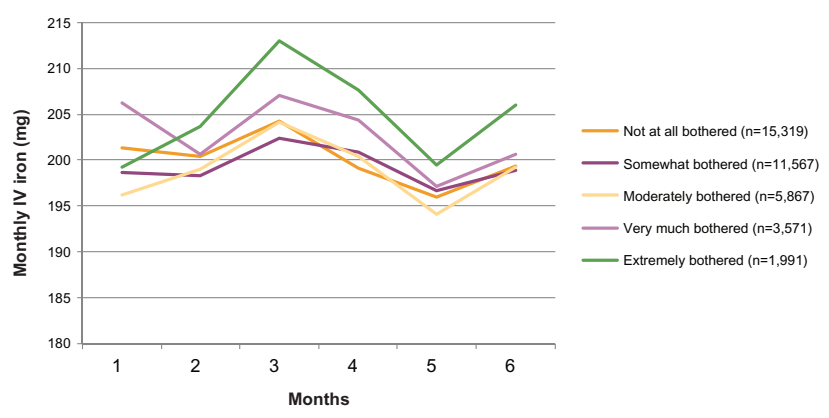

Figure $\mathbf{5}$ Intravenous (IV) iron utilization difference by itchiness score (detailed analysis). particularly among those with a history of infections such as bacteremia and septicemia. The clinically meaningful differences found in IV antibiotic use among patients reporting itchy skin suggest that increasing pruritus correlates with increased infection rates and health care resource utilization, which could have health-related outcomes and economic implications that need to be managed.

While statistically significant differences were observed with clinical laboratory results (phosphorous, corrected calcium, $\mathrm{Ca} * \mathrm{P}, \mathrm{PTH}$, serum ferritin, albumin) across itchiness severity, these differences were not clinically meaningful. A recent study by Makhlough et al, found that PTH level did correlate with itchiness severity, but that other laboratory parameters did not correlate with itchiness. ${ }^{23}$

Patients reporting increasing itchiness had higher use of dialysis medications including IV antibiotics, use of pruritus treatments, and ESA use. For ESA use, we found clinically meaningful differences $(>5,000$ units/month) when patients with severe itchy skin were compared to patients with no itchiness complaints. However, in a previously published study with hemodialysis patients, erythropoietin intake did not correlate with the incidence or intensity of pruritus. ${ }^{24}$ There is some evidence that septicemia is associated with higher ESA use, ${ }^{25}$ and inflammation is associated with increased ESA resistance. ${ }^{26}$ We hypothesize that the results of this study may be due to the constellation of itch symptoms or an ineffective ESA response, but such a hypothesis is beyond the scope of this analysis.

Those affected with increasing itchiness severity were also found to be more likely to miss HD sessions, and missed HD sessions result in long intradialytic intervals that have been associated with increased all-cause, cardiac-related, and infection-related hospitalizations and mortality. ${ }^{27}$ The relationship between intradialytic intervals, missed HD sessions, and pruritus on clinical and economic outcomes warrants further investigation.

These analyses have several limitations. Responses to itchiness severity questions in the KDQOL are self-reported; patients may under- or over-report their true severity of pruritus. Hence, the study is subject to responder and misclassification 
bias. The International Forum for the Study of Itch (IFSI) reported that there is no universal objective measure of itch; however, numerous instruments are being used, some of which have been validated for uremic pruritus, including the visual analogue scale, the numerical rating scale, the Brief Itchiness Inventory, Skindex-10, and the 5-D Itch scale. ${ }^{28,29}$

Second, with a large sample size it is possible that small differences in metrics may be statistically significant without being clinically meaningful. ${ }^{30,31}$ Through the use of adjusted multivariate models, we attempted to account for many of the confounding factors that would further limit generalizability of the associations found in this study.

Third, the analysis of pruritus treatments was complicated because over-the-counter (OTC) medications are self-reported by the HD patients upon arrival at the dialysis clinic. ${ }^{2}$ Therefore, any absent data may represent either no use of OTC pruritus treatments or missing data. Additional analyses are needed with regard to current pruritus therapy management options and related costs, including out-of-pocket costs for OTC medications. In addition, due to the high presence of comorbidities, the possibility of medication-related etiology of pruritus and/or systemic origin beyond the kidney must be addressed.

Fourth, there may be residual confounding from unobserved factors that could not be measured in our data. Such factors may affect our comparison of outcomes between itchiness categories. To control for comprehensive selection of baseline variables, a regression analysis of medication utilization was used. Hence, results must be interpreted with caution. Our study only shows associations, not causality.

Despite these limitations, results of this study indicate a high economic burden on dialysis providers and payers in the largest sample population studied to date. The findings suggest that dialysis-related costs were higher with increasing severity of self-reported itchiness. For example, compared to patients reporting "not at all bothered" by itchiness, the total dialysis-related costs during the 6-month follow-up period were higher with each increasing category of itchiness severity. Major drivers of increased cost were increased use of ESAs, IV antibiotics, and missed sessions across all itchiness subgroups. While economic outcomes were not a focus of this study, these findings spurred additional analyses that will be presented in a future manuscript.

\section{Conclusion}

The clinical burden of pruritus in ESRD patients receiving dialysis may represent a substantially higher burden than previously understood. These patients report poorer qualityof-life measures (both physical and mental), have higher prevalence of comorbid conditions, demonstrate increased medication use, such as ESA and IV antibiotics, and miss more dialysis sessions. The increased clinical burden demonstrated that these patients may represent poorer clinical outcomes and higher economic burden to dialysis facilities and payers. Appropriate assessment of symptomatology and effective control of pruritus is important to improve clinical outcomes and quality of life as well as the related resource utilization and health care dollars in the ESRD patient population.

\section{Acknowledgments}

This study was funded by Mitsubishi Tanabe Pharma Corporation. We thank DaVita Clinical Research (DCR) for providing technical support for this research project. We thank Michele G Scheid and Christopher A Graybill $\mathrm{PhD}$, of DCR for editorial support.

\section{Disclosure}

TCB, AC, and SS are employees of DaVita Clinical Research Inc. KR was an employee of DCR at the time the research was conducted and the manuscript written. VS, MK, and WA are employees of Mitsubishi Tanabe Pharma Corporation. The authors report no other conflicts of interest in this work.

\section{References}

1. Pisoni RL, Wikström B, Elder SJ, et al. Pruritus in haemodialysis patients: International results from the Dialysis Outcomes and Practice Patterns Study (DOPPS). Nephrol Dial Transplant. 2006;21(12): 3495-3505.

2. Mathur VS, Lindberg J, Germain M, et al; ITCH National Registry Investigators. A longitudinal study of uremic pruritus in hemodialysis patients. Clin J Am Soc Nephrol. 2010;5(8):1410-1419.

3. Khanna D, Singal A, Kalra OP. Comparison of cutaneous manifestations in chronic kidney disease with or without dialysis. Postgrad Med J. 2010;86(1021):641-647.

4. Metz M, Ständer S. Chronic pruritus - pathogenesis, clinical aspects and treatment. J Eur Acad Dermatol Venereol. 2010;24(11):1249-1260.

5. Kuypers DR. Skin problems in chronic kidney disease. Nat Clin Pract Nephrol. 2009;5(3):157-170.

6. Wikström B, Gellert R, Ladefoged SD, et al. Kappa-opioid system in uremic pruritus: multicenter, randomized, double-blind, placebo-controlled clinical studies. J Am Soc Nephrol. 2005;16(12): 3742-3747.

7. Kumagai H, Ebata T, Takamori K, Muramatsu T, Nakamoto H, Suzuki H. Effect of a novel kappa-receptor agonist, nalfurafine hydrochloride, on severe itch in 337 haemodialysis patients: a Phase III, randomized, double-blind, placebo-controlled study. Nephrol Dial Transplant. 2010;25(4):1251-1257.

8. Gunal AI, Ozalp G, Yoldas TK, Gunal SY, Kirciman E, Celiker H. Gabapentin therapy for pruritus in haemodialysis patients: a randomized, placebo-controlled, double-blind trial. Nephrol Dial Transplant. 2004;19(12):3137-3139.

9. Manenti L, Vaglio A, Costantino E, et al. Gabapentin in the treatment of uremic itch: an index case and a pilot evaluation. J Nephrol. 2005;18(1): 86-91. 
10. Naini AE, Harandi AA, Khanbabapour S, Shahidi S, Seirafiyan S, Mohseni M. Gabapentin: a promising drug for the treatment of uremic pruritus. Saudi J Kidney Dis Transpl. 2007;18(3):378-381.

11. Razeghi E, Eskandari D, Ganji MR, Meysamie AP, Togha M, Khashayar P. Gabapentin and uremic pruritus in hemodialysis patients. Ren Fail. 2009;31(2):85-90.

12. Aperis G, Paliouras C, Zervos A, Arvanitis A, Alivanis P. The use of pregabalin in the treatment of uraemic pruritus in haemodialysis patients. J Ren Care. 2010;36(4):180-185.

13. Solak Y, Biyik Z, Atalay H, et al. Pregabalin versus gabapentin in the treatment of neuropathic pruritus in maintenance haemodialysis patients: a prospective, crossover study. Nephrology (Carlton). 2012;17(8):710-717.

14. Marquez D, Ramonda C, Lauxmann JE, et al. Uremic pruritus in hemodialysis patients: treatment with desloratidine versus gabapentin. J Bras Nefrol. 2012;34(2):148-152.

15. Weisbord SD, Fried LF, Arnold RM, et al. Prevalence, severity, and importance of physical and emotional symptoms in chronic hemodialysis patients. J Am Soc Nephrol. 2005;16(8):2487-2494.

16. Kini SP, DeLong LK, Veledar E, McKenzie-Brown AM, Schaufele M, Chen SC. The impact of pruritus on quality of life: the skin equivalent of pain. Arch Dermatol. 2011;147(10):1153-1156.

17. Narita I, Alchi B, Omori K, et al. Etiology and prognostic significance of severe uremic pruritus in chronic hemodialysis patients. Kidney Int. 2006;69(9):1626-1632.

18. Chen HY, Chiu YL, Hsu SP, et al. Elevated C-reactive protein level in hemodialysis patients with moderate/severe uremic pruritus: a potential mediator of high overall mortality. QJM. 2010;103(11):837-846.

19. Leggat JE, Orzol SM, Hulbert-Shearon TE, et al. Noncompliance in hemodialysis: predictors and survival analysis. Am J Kidney Dis. 1998;32(1):139-145.

20. Hays RD, Kallich JD, Mapes DL, Coons SJ, Carter WB. Development of the kidney disease quality of life (KDQOL) instrument. Qual Life Res. 1994;3(5):329-338.

21. Lowrie EG, Lew NL. Commonly measured laboratory variables in hemodialysis patients: relationships among them and to death risk. Semin Nephrol. 1992;12(3):276-283.
22. Mapes DL, Lopes AA, Satayathum S, et al. Health-related quality of life as a predictor of mortality and hospitalization: the Dialysis Outcomes and Practice Patterns Study (DOPPS). Kidney Int. 2003;64(1):339-349.

23. Makhlough A, Emadi N, Sedighi O, Khademloo M, Bicmohamadi AR. Relationship between serum intact parathyroid hormone and pruritus in hemodialysis patients. Iran J Kidney Dis. 2013;7(1):42-46.

24. Szepietowski JC, Sikora M, Kusztal M, Salomon J, Magott M, Szepietowski T. Uremic pruritus: a clinical study of maintenance hemodialysis patients. J Dermatol. 2002;29(10):621-627.

25. Nissenson AR, Dylan ML, Griffiths RI, Yu HT, Dubois RW. Septicemia in patients with ESRD is associated with decreased hematocrit and increased use of erythropoietin. Clin J Am Soc Nephrol. 2006;1(3): 505-510.

26. Icardi A, Paoletti E, De Nicola L, Mazzaferro S, Russo R, Cozzolino M. Renal anaemia and EPO hyporesponsiveness associated with vitamin D deficiency: the potential role of inflammation. Nephrol Dial Transplant. 2013;28(7):1672-1679.

27. Foley RN, Gilbertson DT, Murray T, Collins AJ. Long interdialytic interval and mortality among patients receiving hemodialysis. $N$ Engl J Med. 2011;365(12):1099-1107.

28. Ständer S, Augustin M, Reich A, et al. Pruritus assessment in clinical trials: consensus recommendations from the International Forum for the Study of Itch (IFSI) special interest group scoring itch in clinical trials. Acta Derm Venereol. 2013;93(5):509-514.

29. Elman S, Hynan LS, Gabriel V, Mayo MJ. The 5-D itch scale: a new measure of pruritus. Br J Dermatol. 2010;162(3):587-593.

30. Man-Son-Hing M, Laupacis A, O'Rourke K, et al. Determination of the clinical importance of study results. J Gen Intern Med. 2002;17(6): 469-476.

31. Hays RD, Woolley JM. The concept of clinically meaningful difference in health-related quality-of-life research. How meaningful is it? Pharmacoeconomics. 2000;18(5):419-423.

\section{Publish your work in this journal}

The International Journal of Nephrology and Renovascular Disease is an international, peer-reviewed open-access journal focusing on the pathophysiology of the kidney and vascular supply. Epidemiology, screening, diagnosis, and treatment interventions are covered as well as basic science, biochemical and immunological studies. The journal welcomes original research, clinical studies, reviews \& evaluations, expert opinion and commentary, case reports and extended reports. The manuscript management system is completely online and includes a very quick and fair peerreview system, which is all easy to use. Visit http://www.dovepress.com/ testimonials.php to read real quotes from published authors. 